\title{
Actions of Two Bi-Articular Muscles of the Lower Extremity: A Review
}

\author{
Dennis Landin ${ }^{\mathrm{a}, \mathrm{b}}$, Melissa Thompson ${ }^{\mathrm{a}}$, Meghan Reid ${ }^{\mathrm{a}}$
}

\begin{abstract}
The extremities of the human body contain several bi-articular muscles. The actions produced by muscles at the joints they cross are greatly influenced by joint moment arms and muscle length. These factors are dynamic and subject to change as joint angles are altered. Therefore, to more completely understand the actions of such muscles, the angles of both joints must be manipulated. This report reviews investigations, which have explored the actions of two biarticular muscles of the lower extremities (gastrocnemius and rectus femoris) as the joints they cross are moved into various combinations of angles. The findings have both clinical and physical performance ramifications.
\end{abstract}

Keywords: Bi-articular muscles; Lower extremity; Joint angles

\section{Introduction}

Bi-articular muscles are commonly found in the upper and lower extremities of the human body. These muscles generally cross two joints and influence movement at both. The rectus femoris (RF) spans the hip and knee, and the gastrocnemius (GA) crosses the knee and ankle. The actions of these muscles at their primary joints have been known for well over 100 years [1]. The RF is an extensor of the leg, and the GA is a powerful plantarflexor. The descriptions of these particular actions have been relatively unchanged for many years and appear in most anatomy textbooks [2-4]. However, these muscle action descriptions do not consider the influence the second joint may have on the muscle's action at the primary joint, or vice versa [5]. For example, considering the GA action at the ankle, how does the plantarflexion (PF) torque it generates change as the angles of the knee and ankle change? At what

Manuscript accepted for publication February 03, 2016

aSchool of Kinesiology, Louisiana State University, Baton Rouge, LA 70810, USA

${ }^{b}$ Corresponding Author: Dennis Landin, School of Kinesiology, Louisiana State University, Baton Rouge, LA 70810, USA. Email: dlandin@1su.edu

doi: http://dx.doi.org/10.14740/jocmr2478w combination does muscular insufficiency arise? Advances in technology have made it possible to answer questions of this type, resulting in more detailed descriptions of the bi-articular muscles of the extremities. In our previous papers, we discussed in some detail issues such as muscle tissue, joint moments and moment arms [6-8] and their effects on bi-articular muscle actions.

\section{GA}

The GA is one of 14 muscles that act upon the knee, and nine of these, including the GA are bi-articular [5]. However, the GA is only one of these nine that acts on both the knee and ankle, and the others cross the knee and hip. At the knee, the GAs' actions oppose those of the quadriceps femoris, acting synergistically with the other primary knee flexors (biceps femoris, semitendinosus, semimembranosus, gracilis, popliteus, and sartorius). But it also belongs to another group of muscles that cross the ankle. It opposes the action of the dorsiflexors (e.g., tibialis anterior, extensor digitorum longus and hallucis longus) and it is a powerful plantarflexor working with other posterior leg muscles (e.g., soleus, tibialis posterior, flexor digitorum and hallucis longus). PF forces can be quite high. It is estimated that young males can generate PF torque ranging between 1,000 to $1,780 \mathrm{~N}$ [5].

The GA consists of two heads arising from the posterior aspects of the femoral condyles. These merge into a common belly that rides on the proximal half of the sural aspect of the leg. It shares an insertion with the soleus on the calcaneus via the achilles tendon. The GA and soleus are collectively referred to as the triceps surae and the innervation is supplied by the tibial nerve entering the proximal segments of the muscles. These two muscles provide approximately $80 \%$ of force of PF, which is a principal component to a large portion of the gait cycle and essential to nearly all forms of human locomotion. Di Nardo et al [9] explored the GA's role in PF using sEMG to measure activation patterns in the lateral portion of the GA when walking. These investigators found that the lateral portion of the GA was active in the stance phase when transitioning from flat foot contact to toe-off and then again in the final portion of the swing phase.

Riemann et al [10] investigated the GA's contribution to the stability of the ankle across three ankle angles. These in- 


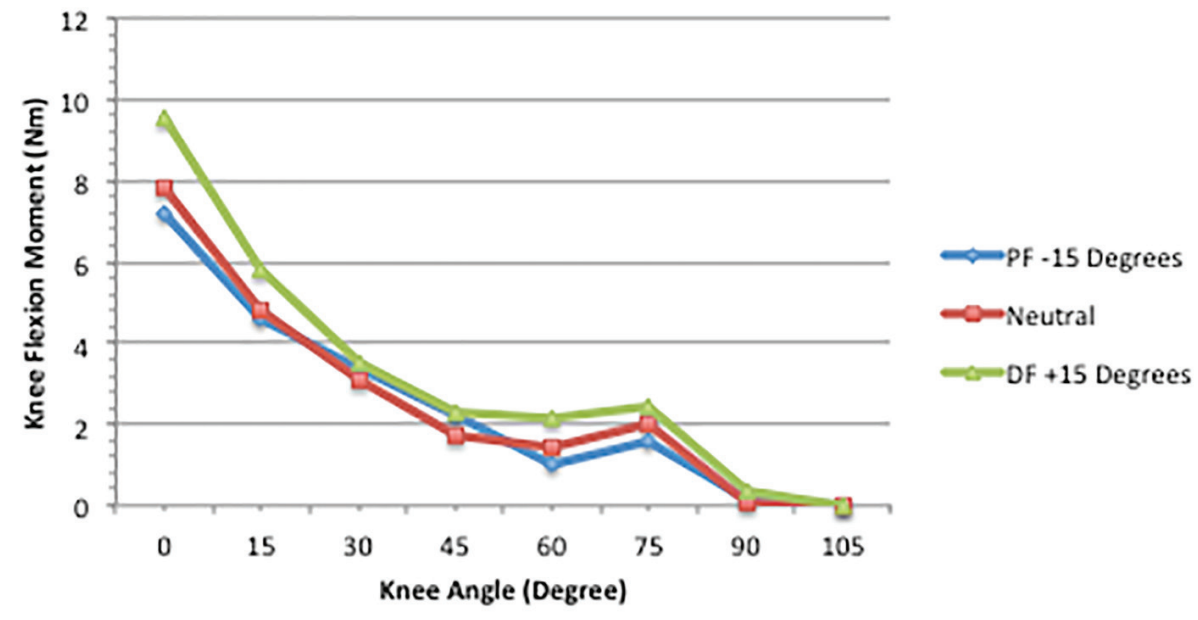

Figure 1. MM across knee and ankle joint combinations [8].

vestigators measured the stiffness values of the GA as the subject's ankles were passively moved from $10^{\circ}$ of dorsiflexion through the neutral position and into $10^{\circ}$ of $\mathrm{PF}$, while the knee was alternately positioned at 0 and $90^{\circ}$. The results showed that the $0^{\circ}$ knee angle produced higher stiffness values across the three ankle positions than the $90^{\circ}$ knee angle. This shows that both joints influence the forces the GA produces. The stiffness index increased as the ankle moved from PF to dorsiflexion and actually began early in the neutral position.

In our initial project with the GA [8], we investigated how the knee and ankle joint angles interacted in the development of knee joint flexion moments. We used 17 subjects (10 females and seven males) in this project and measured the knee flexion moments produced by the GA at 24 knee and ankle joint positions. Knee angles of 0 (anatomical position), 15, 30, $45,60,75,95$, and $105^{\circ}$ ( $\mathrm{Li}$ et al [8] labeled the knee angles from the anatomical position as follows: 180, 165, 150, 135, $120,105,90$, and $75^{\circ}$. In Landin et al [7], they used the more common clinical sequence starting with 0 for the anatomical position, and then progressing through $15,30,45,60,75,90$, and $105^{\circ}$ of knee flexion. The Li et al sequence was converted to avoid confusion.) were crossed with three ankle positions (neutral, $15^{\circ}$ dorsiflexed and plantarflexed). A Biodex System 3 dynamometer controlled the knee angles and removable casts were used to hold the ankle in each of its three positions.

Three dependent measures were of interest: 1) passive moment (PM), which was the joint moment without stimulation, and obtained by finding the mean of the joint moment before and after the stimulation period; 2) maximum moment (MM), which was the joint moment during the stimulation; and 3) stimulated moment (SM), which was the joint moment induced by the stimulation and was obtained by taking the difference between the MM and PM. Except where unique results were obtained, this review will deal with the MM findings since it represents the moments most applicable to the activities of daily living.

Results (Fig. 1) showed that the MM reduced linearly as knee flexion increased from the anatomical $\left(0^{\circ}\right)$ position. This reduction became greater as the ankle joint moved from dorsi- flexion to PF. Moving the ankle from dorsiflexion to the neutral position induced a greater reduction than the move from neutral to PF. Furthermore, MM production was influenced more by ankle joint changes with the knee between 0 and $30^{\circ}$ compared to the lesser degrees of knee angle.

The most striking observation was that the knee flexion moment decreased approximately $50 \%$ as knee flexion moved from 0 to $30^{\circ}$. The reduction in the MM slowed across the $45-75^{\circ}$ knee angles. When the knee was flexed beyond $75^{\circ}$, the stimulation had little effect on the knee flexion moment. In other words, the GA's greatest contribution to knee flexion occurred with the knee in full extension. This was observed across the three ankle joint angles.

In a recent follow-up to the Li et al's [8] paper, Landin et al [6] used similar methods to measure the GA production of a PF joint moment. Figure 2 illustrates the principal finding regarding the MM. Significant effects were obtained for the knee. A fully extended knee $\left(0^{\circ}\right)$ and a dorsiflexed ankle $\left(+15^{\circ}\right)$ created the highest $(24.95 \pm 10.1 \mathrm{Nm})$ torque values, while the $105^{\circ}$ knee angle and a plantarflexed ankle $\left(-15^{\circ}\right)$ produced the smallest values $(13.02 \pm 4.9 \mathrm{Nm})$. Li et al [8] reported that the GA produced the greatest knee flexion moment in the same joint combination, although the force was not as high (approximately $9.5 \mathrm{Nm}$ ), as we noted for PF (approximately $25 \mathrm{Nm}$ ). We contend that this is most likely due to the disparity in the mechanical advantage of the knee and ankle lever systems. At the knee, the GA works in a thirdclass lever, which produces little mechanical advantage. At the ankle, however, the GA is part of a second-class lever, which has considerable mechanical power [2]. Based on our results and those of $\mathrm{Li}$ et al [8], it is the $0^{\circ}$ knee position and a dorsiflexed ankle that creates the optimal length for the GA to work at either joint. It is apparently the mechanical properties of the lever system that causes the disparity in the GA's knee and ankle joint moments.

Regarding the lowest torque, the results reported in our more recent paper are somewhat in contrast to Li et al [8]. Their results showed that flexing the knee to $90^{\circ}$ or more, regardless of the ankle position, did not significantly reduce torque 


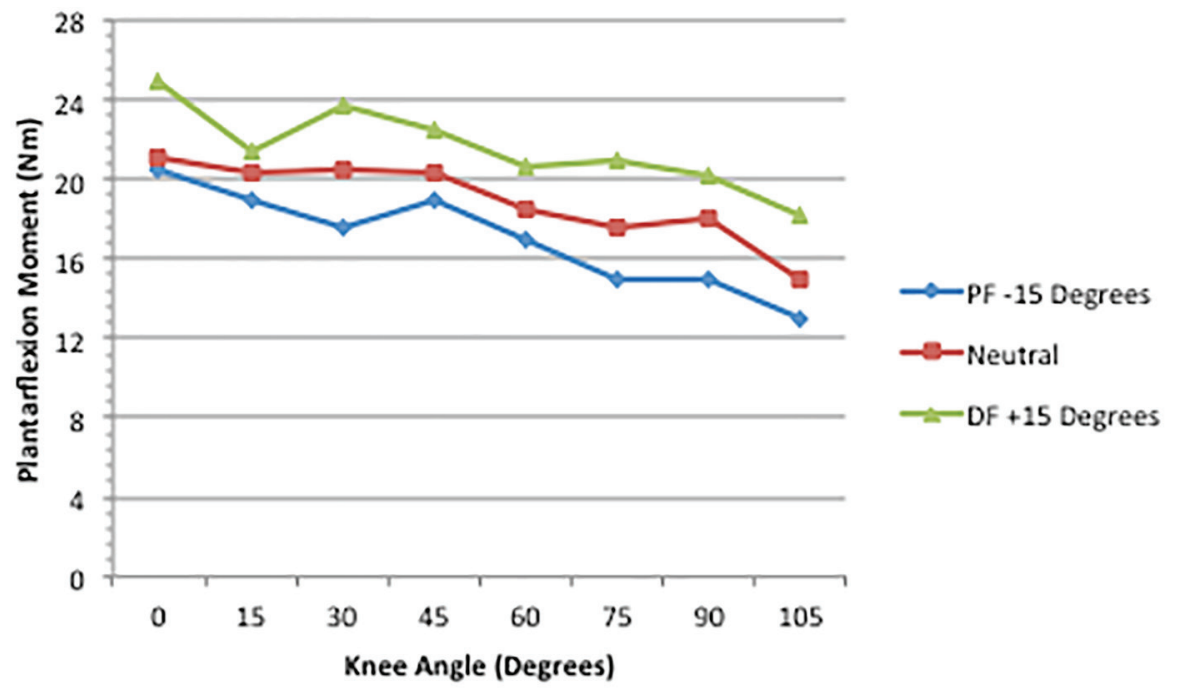

Figure 2. Maximum plantarflexion moment across joint combinations [6].

any further. More precisely, the knee flexion moment did not significantly decrease as the knee was flexed to $105^{\circ}$ with the ankle plantarflexed, neutral, or dorsiflexed. In our more recent project [6], we found that the PF joint moment decreased linearly from the $0 /+15$ joint combination, through the $105 /-15$ combination, at which point the GA was in its most shortened position. It is interesting to note that even in the 105/-15 position, the PF MM (13.02 Nm) exceeded the knee MM reported by $\mathrm{Li}$ et al [8] at the $0 /+15$ position. The GA produced a greater $\mathrm{PF}$ torque in the position of shortest length than it produced for knee flexion in its most elongated position. As argued above, this discrepancy is most likely due to the mechanical properties of a second-class compared to a third-class lever. This lever effect also likely explains the fact that $\mathrm{Li}$ et al found the smallest knee flexion moment before reaching the shortest position for the GA. The mechanical properties of a third-class lever may have rendered the GA insufficient before it reached its shortest length, whereas the second-class lever for the GA at the ankle allowed it to generate a PF torque force throughout the range motion used in these studies. Further research using knee flexion angles beyond $105^{\circ}$ may provide further information on this issue.

Li et al [8] argued that their findings were clinically important due to the GA's perceived role in knee joint stability during various lower extremity movements. Following knee joint traumas such as ACL ruptures, the GA's synergistic work with the quadriceps assists in stabilizing the knee during weight bearing motions [11]. Their results suggested that flexed knee positions $\left(>30^{\circ}\right)$, where the GA has less influence should be avoided, particularly in the early stages of rehabilitation. Rehabilitation exercises that maintain the knee in a position of stability $\left(<30^{\circ}\right)$ appear to be best. Maintaining this knee angle while stimulating co-contraction of all the dynamic stabilizers at the knee, which includes the GA, enhances stability through increased tibiofemoral joint compression, joint stiffness, and proprioceptive influences as suggested by Wilk et al [12].

The clinical implications of Landin et al [6] suggest that having a patient seated at the end of an examination table with the foot subject to gravity, while useful for evaluating the triceps surae, is a poor position from which to assess the GA as a plantarflexor. This position places the knee at greater than $60^{\circ}$ of flexion and maintains a somewhat plantarflexed foot, both of which lead to significant declines in GA torque production. Even if the clinician holds the patient's ankle in a dorsiflexed position, the flexed knee will still mask the GA's true strength. Manual testing of the GA in isolation should be performed, whenever possible, with the knee extended and the ankle dorsiflexed to potentially elicit the maximum PF torque from the GA.

The recent work with the GA reveals that the knee angle is the main factor in the development of both a knee flexion and PF moment of the GA. The GA is a much more powerful plantarflexor than it is a knee flexor, due largely to the mechanical advantage of a second-class lever. However, it is also possible that the 30 total degrees of the ankle ROM used in Li et al [8] and Landin et al [6] was too restrictive. Total ankle ROM (dorsiflexion through PF) can approach $85^{\circ}$ [5]. Moving the ankle closer to the extreme ends of the ROM may have altered the findings. There are difficulties with attaining the full ankle ROM. The dorsiflexion range is reduced as the knee moves closer to full extension, and therefore, cannot be achieved in all joint combinations. Still, future investigations should employ as much of the ankle ROM as possible.

\section{RF}

The RF is the sole bi-articular muscle of the quadriceps group. A direct head and a reflected head form its proximal attachments. The direct attachment arises from the anterior inferior iliac spine (AIIS) of the pelvis, while the reflected attachment emerges from the rim of the acetabulum and the fibrous capsule of the hip joint. The two proximal attachments quickly blend into a common belly that coalesces into the quadriceps 


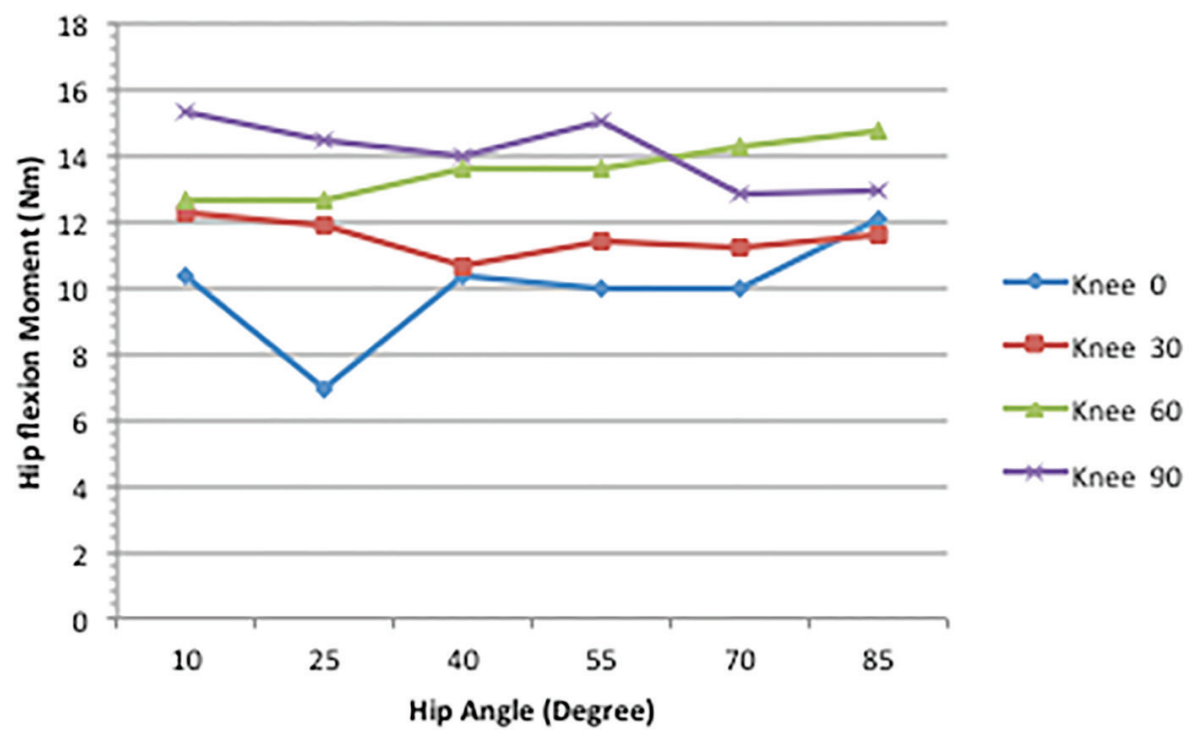

Figure 3. Maximum hip flexion moment across joint combinations [7].

tendon and, in conjunction with the vasti muscles, then inserts on the tibial tuberosity [1-4].

The knee extension work of the quadriceps group is widely known and well documented [1], with a $60-90^{\circ}$ knee angle appearing optimal [13]. Although the function of the RF as a hip flexor is largely unknown, anatomists have traditionally included it with the hip flexor group [2,3]. At the hip, the RF works in a third-class lever, meaning that the application of the force lies between a proximal fulcrum and a distal resistance. As such, the hip is specialized for range and speed of motion [14], but high demands are placed on the muscles moving the lever. Consequently, the RF is heavily involved in all swinging actions of the lower extremity. In Landin et al [6], we cited research with kicking for an often used example. Hsu et al [15] described this action as placing unique demands on the RF for it is the only sport-related movement that requires substantial hyperextension of the hip at the outset. This passively stretches the RF, then while assisting (to some unknown extent) with the initial hip flexion motion, the RF also quickly becomes involved with extending the leg. As a result, the RF is working simultaneously across two joints and becomes subject to distal strains or tears.

From a clinical perspective, the role of RF in hip flexion pertains to muscle imbalance issues. Muscles can react to stress different ways, and an injury, volitional inactivity, or both can produce stress. Postural muscles tend to tighten, while their antagonistic muscles (the phasic group) tend to weaken. When postural muscles (e.g., iliopsoas, RF, and quadratus lumborum) around the pelvis tighten, the phasic muscles (e.g., rectus abdominis, gluteals, vastus lateralis, medialis, and intermedius) weaken and imbalances arise [16].

Janda [17] coined the phrase "lower crossed syndrome" (LCS) to describe a muscle imbalance across the pelvis. In LCS, the gluteals and abdominals lengthen and weaken, while the iliopsoas and RF shorten and tighten. This often leads to an anterior pelvic tilt, which in turn causes a variety of musculo- skeletal maladies and poor posture. What makes the LCS particularly troublesome is that sitting, even with good posture, creates conditions favorable to its development. Furthermore, rehabilitation protocols following a variety of lower extremity injuries strengthen the RF and the three vasti muscles are a primary focus of the exercises. Quadriceps strength is a key element in making the knee joint more stable and in slowing the progression of osteoarthritis, particularly in partially menisectomized knees [18]. However, since the RF is one of the postural muscles that tend to tighten, the potential exists for an exacerbation of a muscle imbalance and LCS. If the RF plays a significant role in hip flexion, then increasing its strength in a quadriceps regimen presents a quandary. Should the RF not substantially contribute to hip flexion, then the use of quadriceps protocols in rehabilitation can be employed without concern for creating or exacerbating an existing LCS.

In our project with the RF and hip flexion [7], we used 16 female volunteers from a university undergraduate population. The equipment and procedures generally followed the descriptions provided in previous sections. In this project, the Biodex chair controlled hip angles and the removable casts controlled the knee angles. Six hip angles $\left(85,70,55,40,25\right.$, and $\left.10^{\circ}\right)$ were crossed with four knee angles ( 0 the anatomical position, 30,60 , and $90^{\circ}$ ) to create 24 joint combinations. At the hip, the $85^{\circ}$ (upright) and the $10^{\circ}$ (reclined) positions represented the limits of the Biodex chair.

Figure 3 displays the MM of the hip flexion function of the $\mathrm{RF}$ across the various joint combinations. The knee produced the only significant effect on the hip flexion moment of the RF. As knee flexion increased from 0 to $90^{\circ}$, the hip flexion moment rose from 10.44 to $15.3 \mathrm{Nm}$. This can be seen on Figure 3 at the $10^{\circ}$ hip angle.

An intriguing result of this project was the contribution of the PM to the MM. As can be seen in Figure 4, a large portion of the MM was produced by the PM. We reported that this ranged from $74 \%$ to $78 \%$ across the joint combinations. Con- 


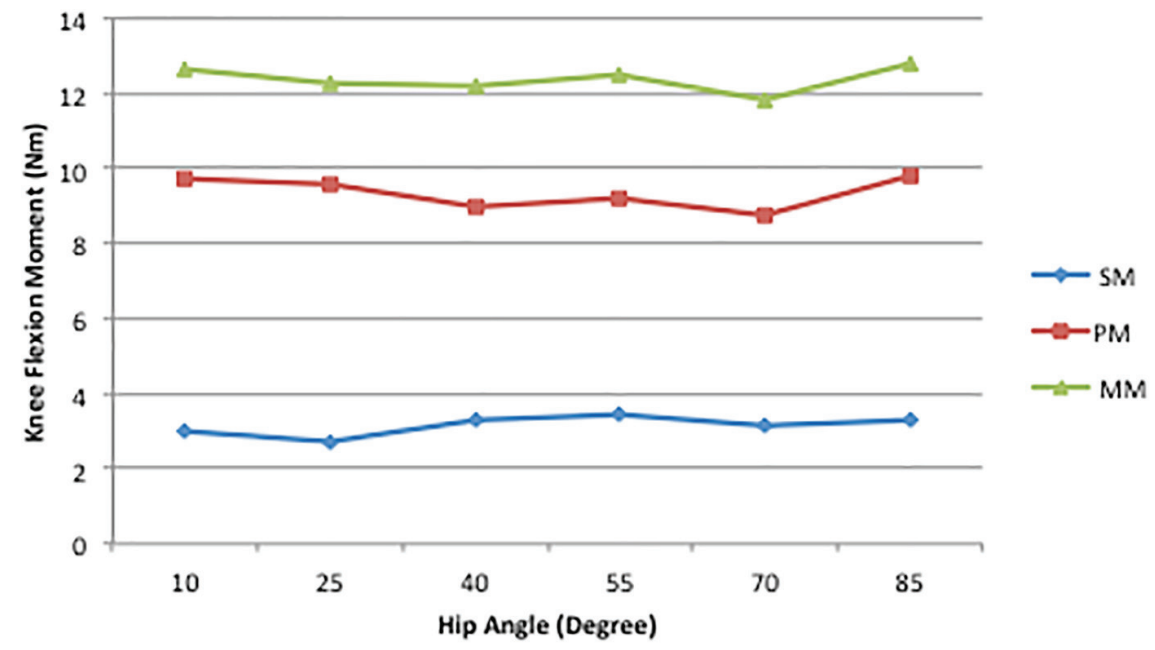

Figure 4. Mean hip flexion SM, PM, MM values for all knee angles at each hip angle [7].

sequently, the stimulation, as shown by the SM line in Figure 4 contributed little to the MM. The hip is so thoroughly supported by various muscle and connective tissue components that in a passive state simply extending the hip can create a substantial hip flexion torque. Moving the hip to the $10^{\circ}$ position used in Landin et al [7] may not have produced an optimal length [19] for the RF during stimulation, but clearly created tension in the other soft tissue structures surrounding the joint. These data show that contraction of the RF accounts for approximately $25 \%$ of the total hip flexion torque, indicating that chronic shortening of the phasic muscles and related fascial components can, even without strengthening of the RF, disrupt the postural/phasic muscle balance. This finding makes clear how the LCS described by Janda [17] can be problematic. One avenue of future work would be investigating the effects stretching routines, either chronic or acute, have on the PM. Shortening of the myofascial components surrounding the hip has been discussed in the literature for some time, and findings from over 20 years ago show that elongating these tissue elements can improve gait [20]. Furthermore, single bouts of stretching have a deleterious effect on power production [21], so there may be an effect on a PM, but it would not necessarily be detrimental regarding LCS issues.

A recent study of muscle activation patterns during gait showed that the RF displays high activity at the start of the swing phase and at stance phase initiation [9]. Our results [7] do not contradict these, but rather indicate that, at the hip angles we used, contraction of the RF does not contribute much to hip flexion. Schadel et al [22] demonstrated that considerable variability in hip extension exists between runners. Motion analysis of 14 elite runners performing a treadmill run at 21 $\mathrm{km} / \mathrm{h}$ showed an average hip extension of $-11.7^{\circ}$, with a range of about $20^{\circ}$. Due to limits of the Biodex chair we were unable to extend the hip beyond $+10^{\circ}$, which is just shy of the anatomical position. A second direction for future work should include hip extension angles that approximate those reported by Schadel et al [22]. It may well be that, since the 10/90 hip and knee position used in our project may have elongated the RF beyond its optimal length, it seems unlikely that greater lengthening would be beneficial. Still, further investigation is needed.

\section{References}

1. Pickering T, Howden R. Muscle. Grays' Anatomy, 15th ed., 1901.

2. Salmons SM. Muscle. In: Williams PL, Bannister LH, Berry MM, Collins P, Dyson M, Dussek JE, Ferguson MWJ, editors. Gray's Anatomy. New York: Churchill Livingstone; 1995. p. 737-900.

3. Tortora GJ. Principles of Human Anatomy. Menlo Park, CA: Benjamin/Cummings, 1999.

4. Van De Graaff KM. Human Anatomy. Dubuque, IA: William C. Brown Communications, 1995.

5. Houglum PA, Bertoti DB. Brunnstrom's Clinical Anatomy. Philadelphia, PA: FA Davis; 2012.

6. Landin D, Thompson M, Reid M. Knee and Ankle Joint Angles Influence the Plantarflexion Torque of the Gastrocnemius. J Clin Med Res. 2015;7(8):602-606.

7. Landin D, Thompson M, Reid M. J Athl Enhancement. 2014;3(2).

8. Li L, Landin D, Grodesky J, Myers J. The function of gastrocnemius as a knee flexor at selected knee and ankle angles. J Electromyogr Kinesiol. 2002;12(5):385-390.

9. Di Nardo F, Ghetti G, Fioretti S. Assessment of the activation modalities of gastrocnemius lateralis and tibialis anterior during gait: a statistical analysis. J Electromyogr Kinesiol. 2013;23(6):1428-1433.

10. Riemann BL, DeMont RG, Ryu K, Lephart SM. The Effects of Sex, Joint Angle, and the Gastrocnemius Muscle on Passive Ankle Joint Complex Stiffness. J Athl Train. 2001;36(4):369-375.

11. Kvist J, Gillquist J. Anterior positioning of tibia during motion after anterior cruciate ligament injury. Med Sci Sports Exerc. 2001;33(7):1063-1072.

12. Wilk KE, Arrigo C, Andrews JR, Clancy WG. Rehabilita- 
tion after anterior cruciate ligament reconstruction in the female athlete. J Athl Train. 1999;34(2):177-193.

13. Westing SH, Seger JY. Eccentric and concentric torquevelocity characteristics, torque output comparisons, and gravity effect torque corrections for the quadriceps and hamstring muscles in females. Int J Sports Med. 1989;10(3):175-180.

14. Bunn JW. Scientific Principles of Coaching. Englewood Cliffs: Prentice-Hall, 1972.

15. Hsu JC, Fischer DA, Wright RW. Proximal rectus femoris avulsions in national football league kickers: a report of 2 cases. Am J Sports Med. 2005;33(7):1085-1087.

16. Norris CM. Abdominal muscle training in sport. $\mathrm{Br} \mathrm{J}$ Sports Med. 1993;27(1):19-27.

17. Janda V. Muscles and motor control in low back pain: Assessment and management. In: Twomey LT (Ed.). Physical therapy of the low back. New York: Churchill Livingstone; 1987 . p. 253-278.

18. Sturnieks DL, Besier TF, Hamer PW, Ackland TR, Mills
PM, Stachowiak GW, Podsiadlo P, et al. Knee strength and knee adduction moments following arthroscopic partial meniscectomy. Med Sci Sports Exerc. 2008;40(6):991997.

19. Gordon AM, Huxley AF, Julian FJ. The variation in isometric tension with sarcomere length in vertebrate muscle fibres. J Physiol. 1966;184(1):170-192.

20. Godges JJ, Macrae H, Longdon C, Tinberg C, Macrae $\mathrm{PG}$. The effects of two stretching procedures on hip range of motion and gait economy. J Orthop Sports Phys Ther. 1989;10(9):350-357.

21. Winchester JB, Nelson AG, Landin D, Young MA, Schexnayder IC. Static stretching impairs sprint performance in collegiate track and field athletes. J Strength Cond Res. 2008;22(1):13-19.

22. Schache AG, Blanch PD, Murphy AT. Relation of anterior pelvic tilt during running to clinical and kinematic measures of hip extension. Br J Sports Med. 2000;34(4):279283. 